\title{
WEIBULL DISTRIBUTION AS AN ACTUARIAL RISK MODEL: COMPUTATION OF ITS PROBABILITY OF ULTIMATE RUIN AND THE MOMENTS OF THE TIME TO RUIN, DEFICIT AT RUIN AND SURPLUS PRIOR TO RUIN
}

\author{
Jagriti Das ${ }^{1 *}$, Dilip C. Nath ${ }^{2}$ \\ ${ }^{1}$ Dept Of Statistics, Gauhati University, Assam, India \\ ${ }^{2}$ Administration, Assam University, Assam
}

\begin{abstract}
The Weibull distribution due to its suitability to adequately model data with high degree of positive skewness which is a typical characteristics of the claim amounts, is considered a versatile model for loss modeling in general Insurance. In this paper, the Weibull distribution is fitted to a set of insurance claim data and the probability of ultimate ruin has been computed for Weibull distributed claim data using two methods, namely the Fast Fourier Transform and the 4 moment Gamma De Vylder approximation. The consistency has been found in the values obtained from the both the methods. For the same model, the first two moments of the time to ruin, deficit at the time of ruin and the surplus just prior to ruin have been computed numerically. The moments are found to be exhibiting behavior consistent to what is expected in practical scenario. The influence of the surplus process being subjected to the force of interest earnings and tax payments on the probability of ultimate ruin, causes the later to be higher than what is obtained in the absence of these factors.
\end{abstract}

Keywords: Classical risk model, Numerical integration, Surplus process, Time to ruin

\footnotetext{
* Corresponding author

Email: jagritistat123.das@gmail.com
} 


\section{Introduction}

Weibull distribution has the virtue of being a mathematically tractable model and is versatile in terms of its applications in reliability, life data analysis, actuarial science and others. Apart from being a potential model in survival analysis and reliability engineering, it has a vast domain of other applications (see [41], [27]) The evidence for the use of Weibull distribution in actuarial statistics is found in [25] and [7]. In the reference [7], the Weibull distribution is fitted to a small data set of hurricane losses whereas [6] used it for modeling two illustrious set of published data namely the Danish fire Insurance data and property claim services data.

In a general Insurance company, one of the main challenges of an actuary is to model the uncertainty involved in the claim arrival pattern and the claim severity pattern by means of suitable random variables. Specifically, the uncertainty in the claim arrival pattern is modeled through a counting distribution whereas a continuous distribution is used for modeling the claim severity. This is done with the objective of risk assessment and this in turn forms the foundation on which is dependent the accuracy of many other actuarial quantities associated with pricing of insurance products, premium loading, the probability of ruin, optimum reserves to be maintained and the identification of appropriate deductibles for reinsurance.

The actuaries try making a financial sense of the future by means of modeling the loss through random variables. Loss modeling constitutes a vital aspect of actuarial science. The insurance industry is important to a country's economy for it provides a means of spreading the financial losses arising out of these risks over a large number of people.

The use of a probability model for explaining the uncertainty in claim severity or the loss amounts; when seen from a perspective of an insurance company, is called loss modeling in general insurance. The reference [25] entails good details on the subject of loss modeling. One of the common characteristic of the data arising from the general insurance sector is the existence of high positive skewness which makes distributions such as Lognormal, Pareto, Gamma, Weibull and Burr, potential candidates for modeling claim severity in general insurance. A very flexible approach to deal with the skewed and fat tails present in financial and economic data is discussed in [24].

One of the classical problems in Actuarial science is the evaluation of the probability of ultimate ruin. Probability of ultimate ruin is a useful measure of the financial risk in an insurance company. It gives some idea on the prospect of insolvency, if any, and hence functions as an early warning system for the guidance of an insurance company. Except for the exponential and the mixture of exponential distributions, for all other loss distributions including Weibull, the probability of ruin can't be obtained analytically and hence, there has been extensive research on a number of computational techniques to obtain this probability. For some of the numerical methods for computing the probability of ultimate ruin. refer to [4], 
[10], [19], [21] and [26].

The moments of the time to ruin, the surplus prior to ruin and the deficit at the time of ruin have academic as well as practical importance in assessing the probable chance for insolvency of the insurance company. The surplus prior to ruin guides the insurance company on the precautionary measures to be taken by it in terms of maintaining enough reserves to avoid any probable chance of ruin. On the other hand, the primary significance of the deficit at the time of ruin is that it guides the action to be taken for recovery in case of occurrence of ruin for the insurance company.

The classical risk model which underlines the framework within which the actuarial quantities are obtained, does not make any assumption on the possibility of the surplus process being subjected to some realistic constraints like the presence of interest rates and the deduction of taxes. In this work, the computation of the probability of ruin is also done when the surplus process is assumed to be subjected to the influence of both of these factors.

The actuarial quantities under consideration don't have any closed form expressions when the underlying claim severity model is Weibull and hence, the use of numerical methods is made to obtain these quantities.

The real significance of this work lies on the fact that we are dealing with the computation of some important actuarial quantities when the underlying claim severity model is Weibull. These quantities don't have a closed form expressions and the selected numerical algorithms which are used for obtaining approximations to these values pose challenges in terms of control of error and the execution time. These aspects have been dealt with moderate success to arrive at the output which are in turn, very important actuarial quantities and whose importance can be appraised from the discussions as given above.

In this paper, use has been made of Fast Fourier Transform (FFT) to compute the probability of ultimate ruin for the Weibull model and the results thus obtained are compared with those obtained by a simple yet considerably accurate approximation namely the 4 moment Gamma De Vylder's approximation. The first two moments of the time to ruin, deficit at the time of ruin and surplus prior to ruin for Weibull distributed claims have been obtained. The impact of the presence of interest earnings and tax payments on the probability of ultimate ruin has also been investigated.

The contents of the paper are organized in the following manner. The section 1 deals with the introduction. The first subsection under methodology deals with the fitting of the Weibull distribution. The computation of the probability of ultimate ruin through FFT and the 4 moment Gamma De Vylder approximation are respectively presented in subsection 3 and subsection 4 of methodology. The subsection 5 and subsection 6 under methodology respectively deals with the concerned moments and the influence of interest rate and tax 
payments on the probability of ultimate ruin. The section 3 is on results and discussions whereas concluding remarks including the limitations of the work and further extensions is presented in section 4 .

\section{Methodology}

\subsection{Fitting of the weibull distribution}

The cumulative distribution function for the two parameter Weibull distribution is given by

$$
F_{w}(x ; \beta, \theta)=1-\exp \left(-\left(\frac{x}{\theta}\right)^{\beta}\right)
$$

where the positive parameters $\beta, \theta$ are respectively the shape and the scale parameters.

And the corresponding probability density function is given by

$$
f(x)=\frac{\beta}{\theta}\left(\frac{x}{\theta}\right)^{\beta-1} \exp ^{-\left(\frac{x}{\theta}\right)^{\beta}}, x \geq 0, \beta>0, \theta>0
$$

If we consider a sample of " $\mathrm{m}$ " items $d_{1}, d_{2} \ldots \ldots d_{m}$ from the Weibull distribution whose probability density function is given by (2), the log likelihood function can be put in the form

$$
I_{w}=m \log \beta-m \beta \log \theta+(\beta-1) S_{e}-\theta^{-\beta} S_{0}(\beta)
$$

where $S_{e}=\sum_{r=1}^{m} d_{r}, S_{0}(\beta)=\sum_{r=1}^{m} d_{r}^{\beta}$

As it can be intuitively felt, the estimation procedure for Weibull distribution is not easy as it does not have closed form expression for its maximum likelihood estimators. The reference [38] have derived a consistent and closed form estimator for the shape parameter of the three parameter Weibull distribution. In our case, use has been made of the multi parameter Newton Raphson method for estimating the parameters of the Weibull distribution. In the appendix, a very brief introduction to the multi parameter Newton Raphson method in accordance with [39] is given.

We give a brief introduction to the classical risk model which constitutes the basis of the framework within which the probability of ultimate ruin and the other related quantities are defined.

\subsection{Classical risk model}

Let $\{U(t)\}_{t \geq 0}$ denote the surplus process of an insurer as

$$
U(t)=u+c t-S(t)
$$

Where $u \geq 0$ is the initial surplus, $c$ is the rate of premium income per unit time and $\{S(t)\}_{t \geq 0}$ is the aggregate claim process and we have $S(t)=\sum_{i=1}^{M(t)} X_{i}$ where $\{M(t)\}_{t \geq 0}$ is a homogeneous Poisson process with parameter $\lambda, X_{i}$ denotes the amount of the ith claim 
and $\left\{X_{i}\right\}_{i=1}^{\infty}$ is a sequence of iid random variables with distribution function $F$ such that $F(0)=0$ and probability density function $f$. We denote $E\left(X_{1}^{k}\right)$ by $p_{k}$. Also we have $c=$ $\left(1+\theta_{1}\right) \lambda p_{1}$, where $\theta_{1}$ is the security loading factor. $p_{1}$ is the mean of the claim severity distribution.

Let $T_{u}$ denote the time to ruin from initial surplus $u$ so that $T_{u}=\inf \{t: U(t)<0\}$ and define $\psi(u)=\operatorname{pr}\left\{T_{u}<\infty\right\}=1-\vartheta(u)$ and $\psi(u, t)=\operatorname{Pr}\left(T_{u} \leq t\right) . \quad \psi(u)$ is known as the ultimate ruin probability whereas $\psi(u, t)$ is the finite time ruin probability. For a detailed discussion on the classical risk model and the probability of ruin, ( see [20], [32], [28], [2] and [26]).

The underlying model in the classical risk model is the compound Poisson risk model since the inter arrival time of the claim arrival process is assumed to be exponentially distributed. Classical risk model is loaded with many simplification assumptions, some of which are not realistic. But it still constitutes the base of many Mathematical models in Actuarial Science. Also, it can be remarked that determining $\psi(u)$ amounts to solving an integro-differential equation (stated later in the paper) which does not permit explicit solutions to most of the claim severity distributions including Weibull distribution. Therefore bounds and approximations to the ultimate ruin probability $\psi(u)$ are required and two of these approximation methods are under consideration in this paper.

\subsection{Computation of the probability of ultimate ruin through the fast fourier transform}

The Fast Fourier transform (FFT) can be applied for the evaluation of certain quantities of interest in the classical risk theory. For example, it can be used for the evaluation of the probability of ultimate ruin and the quantiles of the aggregate claim amount distribution. The approach through which the FFT is explored to evaluate the probability of ultimate ruin can be further extended to the evaluation of the first moment of the time to ruin in the classical risk model.

In this paper, we have used FFT to compute the probability of ultimate ruin for the claim severity distributed as Weibull. The use of FFT for the evaluation of the probability of ruin is discussed in [13]. Methods of reducing the error that creeps into the use of FFT have been discussed in [22] and [23]. The reference [29] also embodies a detailed discussion on the use of FFT for the evaluation of the probability of ultimate ruin. We illustrate the naïve application of FFT in computing the probability of ultimate ruin with no complexity in terms of further attempts at error reduction.

\subsubsection{The fast fourier transform in ruin theory}

As mentioned in [15], the probability of ultimate ruin satisfies the following integro- 
differential equation

$$
\psi^{\prime}(u)=\frac{\lambda}{c} \psi(u)-\frac{\lambda}{c} \int_{0}^{u} \psi(u-x) d F(x)-\frac{\lambda}{c}\{1-F(u)\}, u \geq 0
$$

The following is a description on the use of FFT to compute the probability of ultimate ruin following the ideas as given in [33]

Equation (5) can also be put in the form of a defective renewal equation (see [30])

$$
\psi(u)=\frac{1}{1+\theta_{1}} \int_{0}^{u} \psi(u-x) d F_{X, e}(x)+\frac{1}{1+\theta_{1}} \bar{F}_{X, e}(u)
$$

where $F_{x, e}(u)$ is the claim severity equilibrium distribution given by

$$
F_{X, e}(x)=\frac{1}{p_{1}} \int_{0}^{x}\{1-F(t)\} d t
$$

$p_{1}$ is the mean of the Weibul distribution.

It has been shown in [30], that a solution to (6) is given by

$$
\psi(u)=1-G(u), G=\sum_{k=0}^{\infty}(1-\phi) \phi^{k} F_{X, e}^{*(k)}
$$

where $\phi=\frac{1}{\left(1+\theta_{1}\right)} F_{X, e}^{*(k)}$ denotes the $k^{t h}$ convolution of the claim severity equilibrium distribution.

The equation (6) involves convolutions of the form $\int_{0}^{u} \psi(u-x) d F_{X, e}(x)$ and this renders us valid scope for the use of FFT for the evaluation of the probability of ultimate ruin

\subsubsection{Fast fourier transform in applied probability}

For a sequence $f_{0}, f_{1}, \ldots . f_{M-1}$, the discrete Fourier transformation is defined as

$$
\zeta_{k}=\sum_{m=0}^{M-1} f_{m} \exp \left(\frac{2 \pi m}{M} k\right), k=0,1, \ldots . M-1
$$

and the original sequence $f_{k}$ can be recovered from $\zeta_{k}$ by the inverse transformation

$$
f_{k}=\frac{1}{M} \sum_{m=0}^{M-1} \zeta_{m} \exp \left(-\frac{2 \pi m}{M} k\right), k=0,1,2, \ldots . M-1
$$

Here $\mathrm{M}$ is some truncation point (see [33])

\section{Discretization}

Typically, claim severity distributions are continuous and since FFT allows only discrete severities as input, discretization of the continuous severity is necessary. To concentrate severity, whose continuous distribution is $F(x)$ on $\{0, \sigma, 2 \sigma, \ldots \ldots\}, \sigma>0$, the central difference approximation might be used. The central difference approximation is given by 


$$
\begin{gathered}
f_{0}=F\left(\frac{\sigma}{2}\right) \\
f_{n}=F\left(n \sigma+\left(\frac{\sigma}{2}\right)\right)-F\left(n \sigma-\left(\frac{\sigma}{2}\right)\right), n=1,2,3, \ldots
\end{gathered}
$$

Where $\sigma$ is a small positive discretization parameters. Likewise, the other modes of discretization are the forward difference discretization given by

$$
f_{n}^{u}=F(n \sigma+\sigma)-F(n \sigma)
$$

and the backward difference discretization given by

$$
f_{n}^{L}=F(n \sigma)-F(n \sigma-\sigma)
$$

The implication of the discretized distribution is that it assigns to the non-negative integer ' $n$ ', a probability mass equal to that assigned by $F$ to the interval

$$
\left(\left(n-\left(\frac{1}{2}\right)\right) \sigma,\left(n+\left(\frac{1}{2}\right)\right) \sigma\right]
$$

Proper choice of the truncation parameter $M$ is to be made so that $\left(f_{0}, f_{1}, \ldots f_{M-1}\right)$ can be used as the input array of the FFT. For the effective use of the FFT, we choose $M$ to be a power of 2.To reduce both the discretization and the aliasing error, we need to choose $M$ and $\sigma$ such that $\bar{F}((M-0.5) \sigma)$ is negligible. (see [22],[23])

To obtain the FFT of $\psi(u)$, we require obtaining the Fourier transform of

$$
G=\sum_{k=0}^{\infty}(1-\phi) \phi^{k} F_{X, e}^{*(k)}
$$

It may be noted that in accordance with theorem (8.15) of [28], $\mathrm{G}$ is the distribution function of the maximal aggregate loss random variable given by

$$
L=Y_{1}+Y_{2}+\cdots .+Y_{K}
$$

where $K$ has a geometric distribution with parameter $\phi=\frac{1}{1+\theta_{1}}$ and each of $Y_{i}(i=$ $1,2, \ldots K)$ has a distribution whose distribution function is given by $F_{X, e}$.

To compute the FFT of $L$ which is the discrete counterpart of the characteristic function, we proceed as follows

$$
\begin{aligned}
\chi_{L}(t) & =E\left(e^{i t L}\right)\left(\chi_{L}(t) \text { is the characteristic function of } L\right) \\
& =E\left\{E\left(e^{i t L} \mid K\right)\right\} \\
& =E\left\{\left(\chi_{Y}(t)\right)^{K}\right\}
\end{aligned}
$$

Here $\chi_{Y}(t)$ is the characteristic function of the random variable $Y$ and the result is obtained by making use of the property that the characteristic function of the sum of $K$ independent random variables is equal to the product of the characteristic function of each of the random variables.

We can caste $\chi_{L}(t)$ as 


$$
\begin{aligned}
\chi_{L}(t) & =E\left(e^{-i^{2} K \log \left(\chi_{Y}(t)\right)}\right) \\
& =E\left(e^{i K\left(-i \log \left(\chi_{Y}(t)\right)\right.}\right) \\
& =\chi_{K}\left(-i \log \left(\chi_{Y}(t)\right)\right)
\end{aligned}
$$

where $\chi_{K}($.$) denotes the characteristic function of K$.

But since $K$ has a geometric distribution with parameter $\phi=\frac{1}{1+\theta_{1}}$, therefore, we can write

$$
\chi_{K}(t)=\frac{1-\phi}{1-\phi e^{i t}}
$$

Hence

$$
\chi_{L}(t)=\chi_{K}\left(-i \log \left(\chi_{Y}(t)\right)\right)=\frac{1-\phi}{1-\phi \chi_{Y}(t)}
$$

To compute the FFT of $G$ from the characteristic function of $L$ as obtained in equation (18), We need to discretize the equilibrium distribution as

$$
\begin{gathered}
f_{e, 0}=F_{x, e}\left(\frac{\sigma}{2}\right) \\
f_{e, n}=F_{X, e}\left(n \sigma+\left(\frac{\sigma}{2}\right)\right)-F_{X, e}\left(n \sigma-\left(\frac{\sigma}{2}\right)\right), n=1,2,3, \ldots
\end{gathered}
$$

Since, we require an approximation to the compound Geometric distribution $G$, we first apply the FFT to the array $\left\{f_{e, 0}, f_{e, 1}, f_{e, 2}, \ldots, f_{e, M-1}\right\}$ to obtain $f f t\left(f_{e}\right)$ and then we calculate the array

$$
g_{f f t}=\frac{1-\phi}{1-\phi f f t\left(f_{e}\right)}
$$

The operations are to be carried point wise. The inverse FFT is then applied to give an array $g=\left\{g_{0}, g_{1}, g_{2}, \ldots g_{M-1}\right\}$

Where $g_{n}$ is an approximation to the mass assigned by $G$ to the interval $\left(\left(n-\left(\frac{1}{2}\right)\right) \sigma,\left(n+\left(\frac{1}{2}\right)\right) \sigma\right]$.

Hence an approximation to $\psi((j+0.5) \sigma)$ is given by

$$
\psi_{j}=1-\sum_{i=0}^{j} g_{i}
$$

It can be noted that in obtaining the probability of ruin through FFT, discretized equilibrium distribution of the corresponding claim severity distribution is required. The computation of the equilibrium distribution $F_{x, e}($.$) of Weibull is shown below.$

\subsubsection{Computing the first order equilibrium distribution for weibull distribution}

From (7), we have the first order equilibrium distribution of Weibull as 


$$
F_{x, e}(x)=\frac{1}{p_{1}} \int_{0}^{x}\{1-F(y)\} d y
$$

Here $F(x)=1-e^{-\left(\frac{x}{\theta}\right)^{\beta}}, x>0, \theta>0, \beta>0 \quad, p_{1}=\theta \Gamma 1+\frac{1}{\beta}$

Hence,

$$
\begin{aligned}
\int_{0}^{x}\{1-F(y)\} d y & =\int_{0}^{x} e^{-\left(\frac{y}{\theta}\right)^{\beta}} d y=\frac{\theta}{\beta} \int_{0}^{x_{1}} e^{-z} z^{\frac{1}{\beta}-1} d z, x_{1}=\left(\frac{x}{\theta}\right)^{\beta} \\
& =\frac{\theta}{\beta} \Gamma \frac{1}{\beta} \text { pgamma }\left(x_{1}, \frac{1}{\beta}, 1\right)
\end{aligned}
$$

where $\operatorname{pgamma}(x, a, s)$ in $\mathrm{R}$ software is given by

$$
\operatorname{pgamma}(x, a, s)=\frac{1}{s^{a} \Gamma a} \int_{0}^{x} y^{a-1} e^{-\frac{1}{s} y} d y
$$

and is the representative of the incomplete Gamma function.

\subsection{The 4 moment gamma de vylder approximation for ruin probability}

The 4 moment Gamma De Vylder approximation is an approximation technique for computing the probability of ruin and was proposed by Burnecki, Mista and Weron [5]. It is based on the De Vylder 's idea to replace the claim surplus process $U(t)$ with another one $\bar{U}(t)$ for which the expression for $\psi(u)$ is explicit.

It needs to be noted that an explicit expression for $\psi(u)$ exists for Gamma distribution and hence for any other distribution, for obtaining an approximation to the probability of ruin under it, the distribution is approximated by a Gamma distribution by matching its moments with those of the Gamma distribution. The surplus process $\bar{U}(t)$ for the gamma distribution is characterized by the four parameters $\left(\bar{\lambda}, \bar{\theta}_{1}, \bar{p}_{1}, \bar{p}_{2}\right)$. So, to implement the 4 moment Gamma De Vylder approximation, the four moments of $\bar{U}(t)$ and $U(t)$, the original surplus process are matched to obtain the following expressions for $\left(\bar{\lambda}, \overline{\theta_{1}}, \bar{p}_{1}, \bar{p}_{2}\right)$

$$
\begin{aligned}
\bar{\lambda} & =\frac{\lambda p_{3}^{2} p_{2}^{3}}{\left(p_{2} p_{4}-2 p_{3}^{2}\right)\left(2 p_{2} p_{4}-3 p_{3}^{2}\right)} \\
\overline{\boldsymbol{\theta}}_{\mathbf{1}} & =\frac{\boldsymbol{\theta}_{\mathbf{1}} \boldsymbol{p}_{\mathbf{1}}\left(\mathbf{2} \boldsymbol{p}_{\mathbf{3}}^{\mathbf{2}}-\boldsymbol{p}_{\mathbf{2}} \boldsymbol{p}_{\mathbf{4}}\right)}{\boldsymbol{p}_{\mathbf{2}}^{\mathbf{2}} \boldsymbol{p}_{\mathbf{3}}} \\
\overline{\boldsymbol{p}}_{\mathbf{1}} & =\frac{\mathbf{3}_{\mathbf{3}}^{\mathbf{2}}-\mathbf{2} \boldsymbol{p}_{\mathbf{2}} \boldsymbol{p}_{\mathbf{4}}}{\boldsymbol{p}_{\mathbf{2}} \boldsymbol{p}_{\mathbf{3}}} \\
\bar{p}_{2} & =\frac{\left(p_{2} p_{4}-2 p_{3}^{2}\right)\left(2 p_{2} p_{4}-3 p_{3}^{2}\right)}{\left(p_{2} p_{3}\right)^{2}}
\end{aligned}
$$

For $\bar{\lambda}$ to exist, we need to have $p_{2} p_{4}<\frac{3}{2} p_{3}^{2}$ which is true for gamma distribution.

When this assumption can't be fulfilled, a simple alternative is given by 


$$
\begin{aligned}
\bar{\lambda} & =\frac{2 \lambda p_{2}^{2}}{p_{1}\left(p_{3}+p_{2} p_{1}\right)} \\
\bar{\theta}_{1} & =\frac{\theta_{1} p_{1}\left(p_{3}+p_{2} p_{1}\right)}{2 p_{2}^{2}} \\
\overline{p_{1}} & =p_{1} \\
\overline{p_{2}} & =\frac{p_{1}\left(p_{3}+p_{2} p_{1}\right)}{2 p_{2}}
\end{aligned}
$$

With these notations, the 4 moment Gamma DeVylder approximation is given by

$$
\psi_{4 M G D V}(u)=\frac{\bar{\theta}_{1}\left(1-\frac{R}{\bar{\alpha}}\right) \exp \left(-\frac{\bar{\beta} R u}{\bar{\alpha}}\right)}{1+\left(1+\bar{\theta}_{1}\right) R-\left(1+\bar{\theta}_{1}\right)\left(1-\frac{R}{\bar{\alpha}}\right)}+\bar{\alpha} \bar{\theta}_{1} \frac{\operatorname{Sin}(\bar{\alpha} \pi)}{\pi} I
$$

where

$$
I=\int_{0}^{\infty} \frac{x^{\bar{\alpha}} \exp (-(x+1) \bar{\beta} u)}{\left[x^{\bar{\alpha}}\left\{1+\bar{\alpha}\left(1+\bar{\theta}_{1}\right)(x+1)\right\}-\operatorname{Cos}(\bar{\alpha} \pi)\right]^{2}+\operatorname{Sin}^{2}(\bar{\alpha} \pi)} d x
$$

and

$$
\begin{aligned}
& \bar{\alpha}=\frac{\bar{p}_{2}}{\bar{p}_{2}-\bar{p}_{1}^{2}} \\
& \bar{\beta}=\frac{\bar{p}_{1}}{\bar{p}_{2}-\bar{p}_{1}^{2}}
\end{aligned}
$$

$R$ is the adjustment coefficient for Gamma surplus process with parameters $\left(\bar{\lambda}, \bar{\theta}_{1}, \bar{p}_{1}, \bar{p}_{2}\right)$

For applying this approximation, the first four moments of the claim severity distribution should exist and since for our fitted Weibull distribution, the first four moments exist, the 4 moment Gamma DeVylder approximation can be applied.The 4 moment Gamma DeVylder approximation is often considered best among the simple approximations for probability of ruin (see [21]).

The computation for $\psi_{4 M G D V}(u)$ denoting the 4 moment Gamma DeVylder approximation for $\psi(u)$ is done using the Xplore software (see [3])

\subsection{The moments of the deficit at the time of ruin, surplus just prior to ruin and the time to}

\section{ruin for weibull distributed claim severity}

The analysis of the distribution of the time to ruin, the surplus just prior to ruin and the deficit at the time of ruin are important in warning the management of any adverse situation related to the insolvency of the insurance company. There has been considerable research interest in these aspects. Lack of analytical expression and no closed form solution makes the identification of these distributions very difficult.

The references [16] and [12] carried investigation focused on the analytical expression on the probability of ruin and the distribution function of the surplus before the time of ruin for the case when the individual claim severity distribution is a combination of exponential 
distribution or a combination of gamma distribution. The references [8], [9], [42] and [36] discussed analytical properties of the distribution of the surplus before the time of ruin, the distribution of the deficit at the time of ruin and their relationship. The references [17] and [18] study the joint distribution of the time of ruin, the Surplus before the time of ruin and the deficit at the time of ruin by considering an expected discounted penalty function involving these three random variables They show that this expected discounted penalty as a function of the initial surplus, is the solution of a certain (defective) renewal equation.

Two non-negative random variables in connection to the time to ruin are the surplus just prior to the time of ruin and the deficit at the time of ruin. While the former is denoted by $U\left(T^{-}\right)$, where $T^{-}$is the left hand limit of $T$, the deficit at the time of ruin $T$ is denoted by $|U(T)|$. Vital to the analysis of these quantities, is the role of the Equilibrium distribution.

The following is extracted from [31] which gives the background and the derivation of the formulae for the moments of the deficit at the time of ruin and the surplus just prior to ruin.

Let $W\left(x_{1}, x_{2}\right), 0 \leq x_{1}, x_{2}<\infty$ be a non negative function. For $\tau \geq 0$, define

$$
\varnothing(u)=E\left\{e^{-\tau T} W\left(U\left(T^{-}\right),|U(T)|\right) I(T<\infty)\right\}
$$

where $I(T<\infty)=1$, if $T<\infty$ and $I(T<\infty)=0$,otherwise.

The quantity $W\left(U\left(T^{-}\right),|U(T)|\right)$ can be interpreted as the penalty at the time of ruin, where the surplus is $U\left(T^{-}\right)$and the deficit is $|U(T)|$. The quantity $\emptyset(u)$ is the expected discounted penalty when $\tau$ is interpreted as a force of interest. Since $\psi(u)=E\{I(T<$ $\infty)\}, \emptyset(u)$ reduces to $\psi(u)$ if $\tau=0$ and $W\left(x_{1}, x_{2}\right)=1$.

The reference [18] show that the function $\emptyset(u)$ satisfies the following defective renewal equation

$$
\varnothing(u)=\frac{\lambda}{c} \int_{0}^{u} \emptyset(u-x) \int_{x}^{\infty} e^{-\rho(y-x)} d F(y)+\frac{\lambda}{c} e^{\rho u} \int_{u}^{\infty} e^{-\rho u} \int_{x}^{\infty} W(x, y-x) d F(y) d x
$$

where $\rho=\rho(\tau)$ is the unique non-negative solution of the equation $c \rho-\tau=\lambda-\lambda \tilde{\mathrm{F}}(\rho)$ and $\tilde{F}($.$) denotes the laplace transform of the function F($.

\subsubsection{The deficit at the time of ruin}

Considering $W\left(x_{1}, x_{2}\right)=x_{2}^{k}$, it is derived in theorem (4.1) of [31]

$$
\begin{aligned}
E\left\{e^{-\tau T}|U(T)|^{k} I(T<\infty)\right\} & =\frac{1}{\beta \psi(u)}\left[k \mu_{k-1}(\rho) \alpha_{k-1}(u, \rho)-\mu_{k}(\rho) \bar{K}(u)\right], k \\
& =1,2,3
\end{aligned}
$$

In the special case, when $\tau=0$, we have

$$
E\left\{|U(T)|^{k} \mid T<\infty\right\}=\frac{p_{k}}{p_{1} \theta_{1}} \frac{\tau_{k}(u)}{\psi(u)}-\frac{p_{k+1}}{(k+1) p_{1} \theta_{1}}, k=1,2,3 \ldots
$$

where $\tau_{k}(u)$ are given by the corollary (3.1) of [31], as 


$$
\tau_{1}(u)=\theta_{1} \int_{u}^{\infty} \psi(x) d x
$$

and for $\mathrm{n}=2,3,4 \ldots$

$$
\tau_{n}(u)=\frac{n p_{1}}{p_{n}} \theta_{1} \int_{u}^{\infty}(x-u)^{n-1} \psi(x) d x-\sum_{j=0}^{n-2}\left(\begin{array}{l}
n \\
j
\end{array}\right) \frac{p_{n-j}}{p_{n}} \int_{u}^{\infty}(x-u)^{j} \psi(x) d x
$$

This formula can be used to obtain the moments of the deficit at the time of ruin.

Putting $k=1$, we have

$$
E\{|U(T)| \mid T<\infty\}=\frac{\tau_{1}(u)}{\theta_{1} \psi(u)}-\frac{p_{2}}{2 p_{1} \theta_{1}}
$$

From [15], we have

$$
E(L)=\int_{0}^{\infty} \psi(x) d x=\frac{p_{2}}{2 \theta_{1} p_{1}}
$$

where $L$ is the maximal aggregate loss random variable (see [4])

Hence, equation (29) can be put in the form

$$
\begin{aligned}
\tau_{1}(u) & =\theta_{1} \int_{u}^{\infty} \psi(x) d x \\
& =\theta_{1}\left\{\frac{p_{2}}{2 p_{1} \theta}-\int_{0}^{u} \psi(x) d x\right\}
\end{aligned}
$$

Therefore, from equation (31), we have

$$
E\{|U(T)| \mid T<\infty\}=\frac{p_{2}}{2 p_{1 \theta_{1}}}\left\{\frac{1}{\psi(u)}-1\right\}-\frac{1}{\psi(u)} \int_{0}^{u} \psi(x) d x
$$

$\int_{0}^{u} \psi(x) d x$ is computed numerically using $\psi(x)$ as obtained by the stable recursive algorithm (see [19])

Similarly

$$
E\left\{|U(T)|^{2} \mid T<\infty\right\}=\frac{p_{2}}{p_{1} \theta_{1}} \frac{\tau_{2}(u)}{\psi(u)}-\frac{p_{3}}{3 p_{1} \theta_{1}}
$$

Now,

$$
\begin{aligned}
& \int_{u}^{\infty}(x-u) \psi(x) d x-\int_{u}^{\infty} \psi(x) d x \\
& =\frac{2 p_{1 \theta_{1}}}{p_{2}}\left[\int_{0}^{\infty}(x-u) \psi(x) d x-\int_{0}^{u}(x-u) \psi(x) d x\right]
\end{aligned}
$$




$$
\begin{aligned}
\tau_{2}(u) & =\frac{2 p_{1} \theta_{1}}{p_{2}} \\
& -\left[\int_{0}^{\infty} \psi(x) d x-\int_{0}^{u} \psi(x) d x\right] \\
& =\frac{2 p_{1} \theta_{1}}{p_{2}}\left\{\frac{1}{2}\left[\frac{p_{3}}{2 \theta_{1} p_{1}}+\left(\frac{1}{2}\right)\left(\frac{p_{2}}{\theta_{1} p_{1}}\right)^{2}\right]-u\left[\frac{p_{2}}{2 \theta_{1} p_{1}}\right]-\int_{0}^{u}(x-u) \psi(x) d x\right\} \\
& -\left\{\frac{p_{2}}{2 \theta_{1} p_{1}}-\int_{0}^{u} \psi(x) d x\right\}
\end{aligned}
$$

The equation (36) is obtained using (32) and the following result

$$
\left.E\left(L^{2}\right)=2 \int_{0}^{\infty} x \psi(x) d x=\frac{p_{3}}{2 \theta_{1} p_{1}}+\left(\frac{1}{2}\right)\left(\frac{p_{2}}{\theta_{1} p_{1}}\right)^{2}\right)(\text { see Gerber [15]) }
$$

In equation (36), $\int_{0}^{u}(x-u) \psi(x) d x$ is computed numerically using $\psi(x)$ as obtained by the stable recursive algorithm.

After a value of $\tau_{2}(u)$ is obtained, it is substituted in (36) to get the 2 nd moment of the deficit at the time of ruin.

\subsubsection{Surplus before the time of ruin}

As derived in equation (5.2) of [31]

$$
\begin{aligned}
E\left\{\{U(T-)\}^{j} \mid\right. & \left.\left.U(T)\right|^{k} I(T<\infty)\right\} \\
& =\frac{p_{k}}{p_{1} \theta_{1}}\left\{\int_{0}^{u} \psi(u-x) x^{j} \bar{F}_{k}(x) d x+\int_{u}^{\infty} x^{j} \bar{F}_{k}(x) d x\right\} \\
& -\left\{\frac{k ! j ! p_{k+j+1}}{(k+j+1) ! p_{1} \theta_{1}}\right\} \psi(u)
\end{aligned}
$$

Moments of $U(T-)$ can be obtained from (38) by putting $k=0$ as $E\left\{\{U(T-)\}^{j} \mid T<\infty\right\}$

$$
=\frac{1}{\theta_{1} \psi(u)}\left\{\int_{0}^{u} \psi(u-x) x^{j} d F_{1}(x)+\int_{u}^{\infty} x^{j} d F_{1}(x)\right\}-\frac{p_{j+1}}{(j+1) p_{1} \theta_{1}}
$$

Note that $F_{n}(x)$ is the $n^{\text {th }}$ order equilibrium distribution function of $F(x)$ and as given in [30], it is defined as

$$
F_{n}(x)=1-\bar{F}_{n}(x)=\frac{\int_{0}^{x} \bar{F}_{n-1}(y) d y}{\int_{0}^{\infty} \bar{F}_{n-1}(y) d y}
$$

where $\bar{F}_{0}(x)=1-F_{0}(x)=\bar{F}(x)$

It can be shown that the mean of the distribution function $F_{n}(x), n=1,2, \ldots$ is given by

$$
\int_{0}^{\infty} \bar{F}_{n}(x) d x=\frac{p_{n+1}}{(n+1) p_{n}}
$$


The first order equilibrium distribution function is denoted by $F_{1}(x)$ which is the same as $F_{X, e}(x)$ as defined in equation (7)

From (39), putting $j=1$, we have

$$
E\{\{U(T-)\} \mid T<\infty\}=\frac{1}{\theta_{1} \psi(u)}\left\{\int_{0}^{u} \psi(u-x) x d F_{1}(x)+\int_{u}^{\infty} x d F_{1}(x)\right\}-\frac{p_{2}}{2 p_{1 \theta_{1}}}
$$

Now the integral $\int_{0}^{u} \psi(u-x) x d F_{1}(x)$ is computed using $\psi(x)$ as obtained by the stable recursive algorithm (see [19]) and $d F_{1}(x)=f_{1}(x) d x$, where $f_{1}(x)$ is the density of the first equilibrium distribution of Weibull which has a known form and $\int_{u}^{\infty} x d F_{1}(x)=$ $\int_{0}^{\infty} x d F_{1}(x)-\int_{0}^{u} x f_{1}(x) d x$

$\int_{0}^{\infty} x d F_{1}(x)$ is the first moment of the first equilibrium distribution of Weibull and is given by

$$
\int_{0}^{\infty} x d F_{1}(x)=\frac{p_{2}}{2 p_{1}}
$$

(see formula (2.6) of [31]) and $\int_{0}^{u} x f_{1}(x) d x$ is computed numerically.

Similarly, putting $j=2$ in equation (39), we have

$$
E\left\{\{U(T-)\}^{2} \mid T<\infty\right\}=\frac{1}{\theta_{1} \psi(u)}\left\{\int_{0}^{u} \psi(u-x) x^{2} d F_{1}(x)+\int_{u}^{\infty} x^{2} d F_{1}(x)\right\}-\frac{p_{3}}{3 p_{1 \theta_{1}}}
$$

$\int_{0}^{u} \psi(u-x) x^{2} d F_{1}(x)$ is computed numerically using $\psi(x)$ as obtained by the stable recursive algorithm and $\int_{u}^{\infty} x^{2} d F_{1}(x)=\int_{0}^{\infty} x^{2} d F_{1}(x)-\int_{0}^{u} x^{2} f_{1}(x) d x$

$\int_{0}^{\infty} x^{2} d F_{1}(x)$ is the second moment of the first equilibrium distribution of Weibull and is given by

$$
\int_{0}^{\infty} x^{2} d F_{1}(x)=\frac{p_{3}}{3 p_{1}}
$$

(see formula (2.6) of [30]) and $\int_{0}^{u} x^{2} f_{1}(x) d x$ is computed numerically. 


\subsubsection{The moments of the time to ruin}

The distribution of the time to ruin is another important quantity related to the probability of ruin. As mentioned in [30], there is no closed form expression developed for the distribution of the time to ruin except in case of Exponential, Mixture of Exponentials and the Erlang group of distributions. Hence, for all other distributions, the moments of the time to ruin can be computed only numerically.

Initial ideas on this aspect can be found in [18] and working on those ideas,[30] presented methods from which explicit solutions for the moments of the time to ruin can be found recursively provided that an explicit solution exist for the ultimate ruin probability. The reference [11] simplified the results of [31] to make them mathematically tractable for numerical computation and used them to calculate the approximate values for the moments of the time to ruin when explicit solutions for the probability of ultimate ruin do not exist. In their numerical computations, values of $\psi(u)$ have been calculated from the stable algorithms described in [10].

The reference [31] shows that the $k^{\text {th }}$ moment of the distribution of the time to ruin is given by

$$
E\left(T^{k}\right)=\frac{\psi_{k}(u)}{\psi(u)}, k=1,2,3 \ldots
$$

where,

$$
\psi_{k}(u)=\frac{k}{\lambda p_{1} \theta_{1}} \int_{0}^{u} \psi(u-x) \psi_{k-1}(x) d x+\vartheta(u) \int_{0}^{\infty} \psi_{k-1}(x)-\int_{0}^{u} \psi_{k-1}(x) d x
$$

Formula (6.2.1) of [31] has been simplified in [11] as

$$
\psi_{1}(u)=\frac{1}{\lambda p_{1 \theta_{1}}}\left\{E(L) \vartheta(u)-\int_{0}^{u} \psi(x) \vartheta(u-x) d x\right\}
$$

where, $\psi_{1}(u)$ can be evaluated using numerical integration in case no closed form expression is available for $\psi(u)$

Similarly, $\psi_{2}(u)$ appearing in [31] has been simplified in [11] as

$$
\psi_{2}(u)=\frac{2}{\lambda p_{1} \theta_{1}}\left\{\frac{E\left(L^{2}\right) \vartheta(u)}{2 \lambda p_{1} \theta_{1}}-\int_{0}^{u} \psi_{1}(x) \vartheta(u-x) d x\right\}
$$

$E(L)$ and $E\left(L^{2}\right)$ appearing in equations (48) and (49) are computed using equations (32) and (37) respectively.

It needs to be noted that explicit expressions for moments of the time to ruin can't be obtained for any claim severity distributions except the Exponential, Mixture of exponentials and Erlang group of distributions. This is because except for these distributions, the integrals appearing in equations equations (48) and (49) can't be solved explicitly. We have used 
Simpson's 1/3rd Rule for numerical integration. The occurrence of error in numerical integration is inevitable and situation gets more complex in case there exists no explicit expression for $\psi(u)$. In [11], numerical integration for the evaluation of $\psi_{1}(u)$ is carried by taking values of $\psi(u)$ as obtained from the algorithm discussed in [10]. In contrast to their approach, we have taken the values of $\psi(u)$ obtained from the Stable Recursive Algorithm mentioned in [19]. These are in turn, used for the numerical integration executed for obtaining $\psi_{1}(u)$ and $\psi_{2}(u)$

\subsection{Probability of ultimate ruin under interest earnings and tax payments for the weibull}

\section{distribution}

The influence of interest earnings and tax payments in the classical risk model has been a theme of recent research. In this context, a remarkable result has been the Albrecher -Hipp tax identity (see [1]). In their framework, tax is paid at a fixed rate $\gamma \in[0,1$ ) whenever the insurer is in profitable position. The modified surplus at time $t$ is written as $U_{\gamma}(t)$ and the corresponding ruin and the non-ruin probabilities are denoted by $\psi_{\gamma}(u)$ and $\vartheta_{\gamma}(u)$ respectively.They derived the following simple formula for $\vartheta_{\gamma}(u)$ assuming that the insurer is in a profitable condition immediately after time.

$$
\vartheta_{\gamma}(u)=[\vartheta(u)]^{\frac{1}{1-\gamma}}
$$

As given in Wei [40], the surplus process at time $t, U_{g}(t)$ satisfies the following stochastic differential equation

$$
d U_{g}(t)=\left\{\begin{array}{l}
C_{1}\left(U_{g}(t) \mathrm{dt}-\mathrm{dS}(\mathrm{t})\right), \text { if } U_{g}(t)<M_{g}(t) \\
C_{2}\left(U_{g}(t) \mathrm{dt}-\mathrm{dS}(\mathrm{t})\right), \text { if } U_{g}(t)<M_{g}(t)
\end{array}\right\}
$$

Where $C_{1}($.$) and C_{2}($.$) are two positive functions and M_{g}(t)=\max ^{m}\left\{U_{g}(t): 0 \leq\right.$ $s \leq t\}$ denotes the running maximum of the Surplus process. Whenever, the surplus is at the running maximum, the company is, according to the terminology of [1], is in a profitable situation. Let $\psi_{g}(u)$ denote the ruin probability for this process as a function of the initial surplus $u$.

In this context an important quantity that is been introduced is $q(x)$ which is a conditional probability, that as the surplus process up crosses the level $x$ for the first time, there is a claim at that instant, $q(x)$ denoting the probability that ruin occurs before the surplus return to level $x$. Also for $x \geq u \geq 0$, let $h(u, x)$ denote the probability the surplus process $\left\{U_{g}(t): t \geq 0\right\}$ having initial value $u$ will reach the level $x$ before possible ruin. Consequently it can be realized that $1-q(x)$ gives the probability that the surplus stays non negative before it returns to the level $x$.

According to the proposition (2.1) of [40], we have 


$$
h(u, x)=\exp \left\{-\int_{u}^{x} \frac{\lambda q(y)}{C_{2}(y)} d y\right\}
$$

Also, it can be noted that $h(u, \infty)=\vartheta_{g}(u)$ thereby giving rise to the corollary (2.1) of $[40]$ as

$$
\begin{gathered}
\vartheta_{g}(u)=\exp \left\{-\int_{u}^{\infty} \frac{\lambda q(y)}{C_{2}(y)} d y\right\} \\
, q(u)=\frac{\vartheta_{\mathrm{g}}^{\prime}(\mathrm{u})}{\vartheta_{\mathrm{g}}(\mathrm{u})} \frac{\mathrm{C}_{2}(\mathrm{u})}{\lambda}
\end{gathered}
$$

Hence once $C_{2}(u)$ is known, $\vartheta_{g}(u)$ can be determined if $q(u)$ is known.

The result that we shall be using has been extracted from [40] considering the special cases $C_{1}(x)=c+\delta x$ and $C_{2}(x)=c+\delta x(1-\gamma(x))$ with $\delta>0$ interpreted as a constant force of interest and $\gamma(x) \epsilon[0,1)$ as a surplus dependent tax rate. For this special case, let $\psi_{\delta, \gamma}(u)$ and $\vartheta_{\delta, \gamma}(u)$ denote the corresponding ruin and non-ruin probabilities respectively

Hence, if $C_{1}(x)=c+\delta x$ and $C_{2}(x)=c+\delta x(1-\gamma(x))$ with $\delta>0$, we have from equation(53)

$$
\vartheta_{\delta, \gamma}(u)=\exp \left\{-\int_{u}^{\infty} \frac{\lambda q(y)}{(c+\delta y)(1-\gamma(y))} d y\right\}
$$

This gives

$$
\psi_{\delta, \gamma}(u)=1-\exp \left\{-\int_{u}^{\infty} \frac{\lambda q(y)}{(c+\delta y)(1-\gamma(y))} d y\right\}
$$

Therefore, as $u \rightarrow \infty$ (56) implies

$$
\psi_{\delta, \gamma}(u) \sim \int_{u}^{\infty} \frac{\lambda q(x)}{(c+\delta x)(1-\gamma(x))} d x
$$

We are primarily concerned with the results of [40] which deals with sub-exponential distributions. Weibull distribution belongs to this category.

By definition, a distribution $F$ on $[0, \infty)$ is said to be subexponential if $\bar{F}(x)=1-$ $F(x)>0$ for all $x>0$ and

$$
\lim _{x \rightarrow \infty} \frac{\bar{F}^{n *}(x)}{\bar{F}(x)}=n
$$

$\bar{F}^{n *}(x)$ denoting the $n^{\text {th }}$ convolution of $\bar{F}(x)$ with itself.

For the computation of the probability of ultimate ruin in the presence of interest earnings and tax payments for sub-exponential distributions, the main result of [40] is cited below:

For a distribution $F$ on $[0, \infty)$ with $\bar{F}(x)>0$ for all $x>0$, define

$$
J_{*}(F)=\operatorname{Sup}\left\{-\frac{\log \bar{F}^{*}(v)}{\log v}: v>1\right\}
$$


with

$$
\bar{F}^{*}(v)=\lim _{x \rightarrow \infty} \operatorname{Sup} \frac{\bar{F}(v x)}{\bar{F}(x)} \text { for } v>1
$$

Main Result: Suppose that the claim size distribution $F$ and its equilibrium distribution $F_{1}$ are subexponential and that $J_{*}(F)$ defined by equation (59) satisfies $1<J_{*}(F) \leq \infty$, then

$$
\psi_{\delta, \gamma}(u) \sim \int_{u}^{\infty} \frac{\lambda \bar{F}(x)}{(c+\delta x)(1-\gamma(x))} d x
$$

For obtaining a slight insight into its proof found in [40], the lemma (4.1) stated herein is used to show that for a sub-exponential distribution $q(x) \sim \bar{F}(x)$.

Hence using this in equation (60), the main result stated in equation (61) is obtained.

We have taken the tax structure as used in [40] for our computation. As a matter in practice, the interest rate is subjected to the choice of investment made by the insurance company and the tax rate is governed by the fiscal policies of the country concerned.

The tax structure used is

$$
\gamma(x)=\left\{\begin{array}{c}
0.10,0<x \leq 10^{4} \\
0.18,10^{4}<x \leq 10^{5} \\
0.30,10^{5}<x \leq 10^{6} \\
0.50, x>10^{6}
\end{array}\right\}
$$

An illustrative value for the rate of interest has been taken as $\delta=0.05$.

\subsubsection{The computation of the probability of ruin for weibull distribution under interest}

\section{earnings and tax payments}

The computation of the Probability of Ruin for Weibull distribution under interest Earnings and Tax Payments is as follows

For the Weibul distribution,

$$
F(x)=1-e^{-\left(\frac{x}{\theta}\right)^{\beta}}, x>0, \theta>0, \beta>0
$$

Therefore from (64),we have

$$
\begin{gathered}
\psi_{\delta, \gamma}(u) \sim \int_{u}^{\infty} \frac{\lambda \bar{F}(x)}{(c+\delta x)(1-\gamma(x))} d x \\
=\int_{u}^{10^{4}} \frac{\lambda}{(c+\delta x)} \frac{e^{-\left(\frac{x}{\theta}\right)^{\beta}}}{\left(\frac{90}{100}\right)} d x+\int_{10^{4}}^{10^{5}} \frac{\lambda}{(c+\delta x)} \frac{e^{-\left(\frac{x}{\theta}\right)^{\beta}}}{\left(\frac{90}{100}\right)} d x+\int_{10^{5}}^{10^{6}} \frac{\lambda}{(c+\delta x)} \frac{e^{-\left(\frac{x}{\theta}\right)^{\beta}}}{\left(\frac{90}{100}\right)} d x \\
+\int_{10^{6}}^{\infty} \frac{\lambda}{(c+\delta x)} \frac{e^{-\left(\frac{x}{\theta}\right)^{\beta}}}{\left(\frac{90}{100}\right)} d x
\end{gathered}
$$

Changing the scale, we have 


$$
\begin{aligned}
\psi_{\delta, \gamma}(u) \sim \frac{\lambda}{0.9} \int_{0}^{10^{4}-u} \frac{e^{-\left(\frac{y+u}{\theta}\right)^{\beta}}}{\{c+\delta(y+u)\}} d y+\frac{\lambda}{0.82} \int_{10^{4}-u}^{10^{5}-u} \frac{e^{-\left(\frac{y+u}{\theta}\right)^{\beta}}}{\{c+\delta(y+u)\}} d y \\
+\frac{\lambda}{0.70} \int_{10^{5}-u}^{10^{6}-u} \frac{e^{-\left(\frac{y+u}{\theta}\right)^{\beta}}}{\{c+\delta(y+u)\}} d y+\frac{\lambda}{0.50} \int_{10^{6}-u}^{\infty} \frac{e^{-\left(\frac{y+u}{\theta}\right)^{\beta}}}{\{c+\delta(y+u)\}} d y
\end{aligned}
$$

This integral is computed numerically using R software [34]

\section{Results and discussions}

Our data comprises of a set of 160000 insurance claim data occurring in between April 2013 to September 2013 obtained from a reputed General Insurance company of India. The data pertains to its motor insurance portfolio covering all its branches in India. No adjustment is made for inflation for the time horizon is narrow and since the exact computation of the intensity parameter is not possible owing to the difficulty in keeping track of the inter arrival time of the claim arrival process, so an illustrative value of the intensity parameter is taken as $\lambda=32.427$. Furthermore, an illustrative value for the security loading factor is taken as $\theta_{1}=$ 0.3. It needs mentioning that without placing much emphasis on the practical essence of the data, we are more interested in using it for illustrative purposes in show casing the use of the Weibull distribution as an Actuarial Risk Model, illustrating its fitting to a set of data and computation of the probability of ultimate ruin under this model and some other actuarial quantities.

Summary statistics of the data is shown in Table 1. It indicates the presence of positive skewness in the data and this renders us valid reason to conclude that Weibull distribution can be a potential model for our data. For fitting the Weibull distribution, we have computed the maximum likelihood estimates of the parameters through multi parameter Newton Raphson method. The ideas for its execution are extracted from [39]. The Gradient and the Hessian matrix for Weibull distribution needed for this purpose are being placed in Appendix. The initial values required for the execution of this algorithm are identified on a trial and error basis. The Convergence criterion for this algorithm is found to occur at 100th iteration. Table 2 shows the estimates of the parameters.

The goodness of fit of the Weibull distribution to the observed data, are at first, assessed through some graphical displays. The histogram of a set of data simulated from Weibull with the values of the parameters taken as those of the estimated values (Figure 2) bears some resemblance with the histogram of the observed data displayed in Figure 1. This to some extent justifies the goodness of fit of the Weibull model to the observed data. The moderate deviation 
of the QQ plot from the straight line passing through the origin further supports this fact. However the two EDF (Empirical Distribution Function) statistics namely the Anderson Darling Statistics and Cramer Von Mises Statistics (see [37] and [6]) and their p values computed through Monte Carlo simulation (see [35] ) indicate lack of fit of the Weibull model to our claim dataset. Table 2 also displays the calculated values of these two EDF statistics along with their p-values. Despite lacking adequate suitability to the dataset, the fitted Weibull model is retained as our model for subsequent computations targeted at determination of the actuarial quantities under consideration.

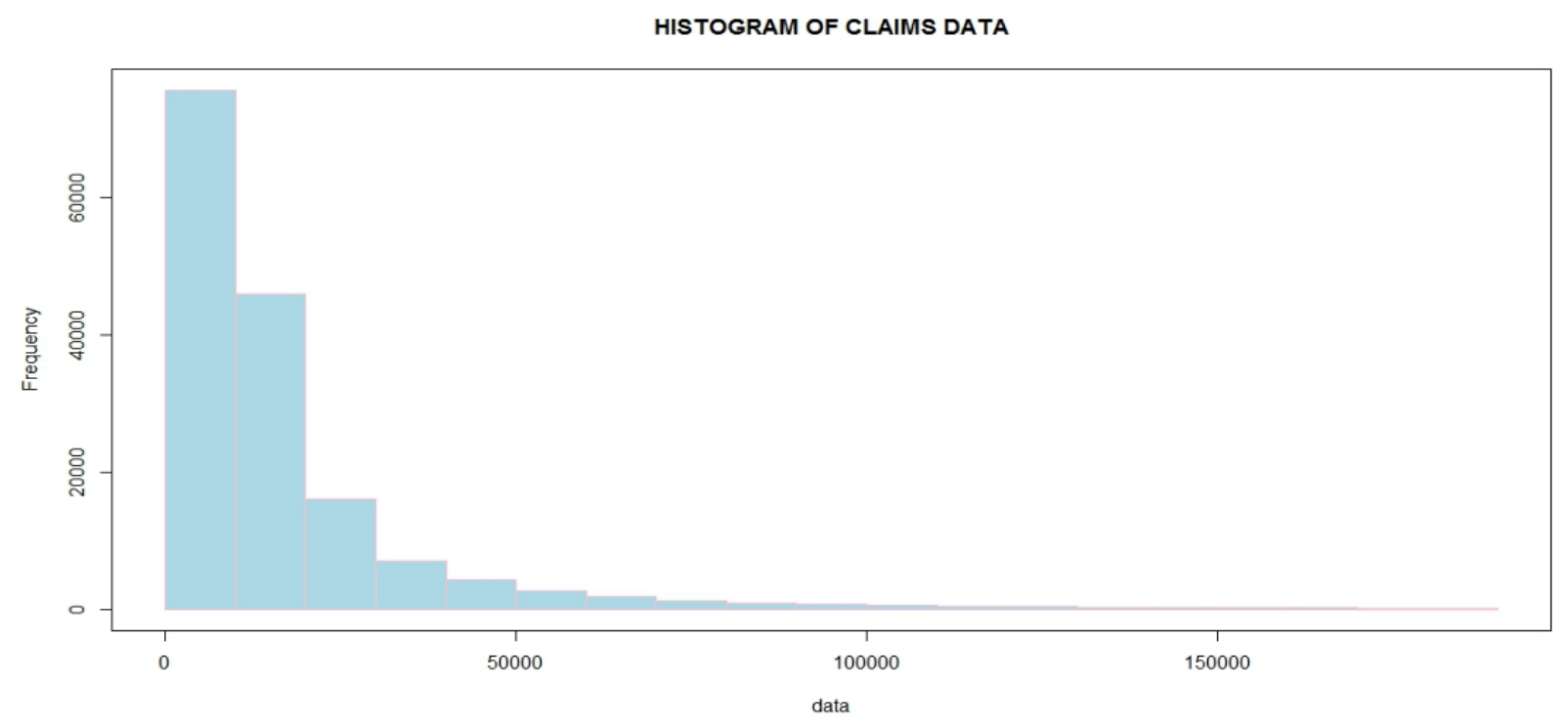

Figure: 1 Histogram of the observed claim data on motor insurance

Table:1 Summary Statistics for the Insurance claim data.

\begin{tabular}{|c|c|c|c|c|c|c|c|c|c|}
\hline $\begin{array}{c}\text { Sample } \\
\text { Size }\end{array}$ & Mean & $\begin{array}{c}\text { Standard } \\
\text { deviation }\end{array}$ & Min & $\begin{array}{c}25 \% \\
\text { Quantile }\end{array}$ & Median & $\begin{array}{c}75 \% \\
\text { Quantile }\end{array}$ & Max & Skewness & Kurtosis \\
\hline 160000 & $1.78834 \mathrm{e}+04$ & 22805.81 & 523 & 6043.00 & 10583.00 & 19374.25 & 188209 & 3.576628 & 18.94972 \\
\hline
\end{tabular}

Table:2 Parameter estimates for the Weibull distribution obtained through the Multi parameter Newton Raphson and the value of the EDF statistics along with their p-values indicated in parentheses.

\begin{tabular}{|c|c|}
\hline Parameter & Estimate \\
\hline$\hat{\theta}$ & 18058.838357 \\
$\hat{\beta}$ & 1.0196673 \\
\hline Anderson Darling statistics & $4123.742(0.04)$ \\
\hline Cramer Von statistics & $655.1592(0.07)$ \\
\hline
\end{tabular}




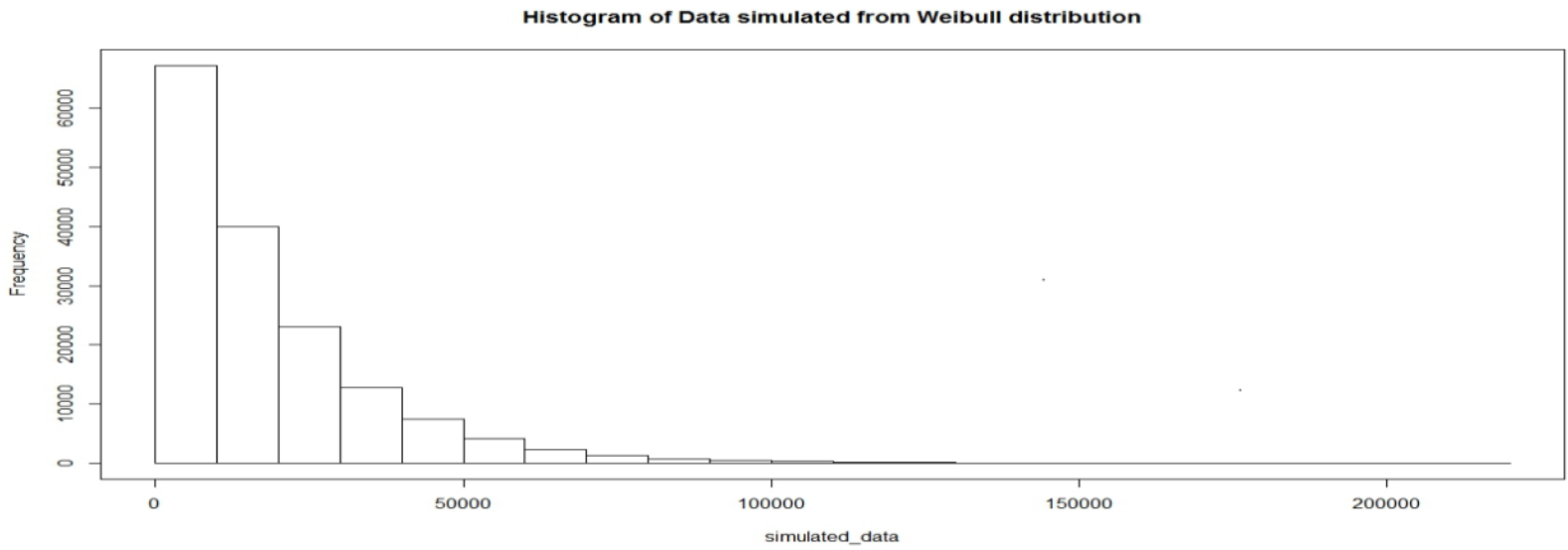

Figure: 2 Histogram for a data set simulated from the Weibull Distribution with $\hat{\theta}=18058.838357$ and $\hat{\beta}=1.0196673$

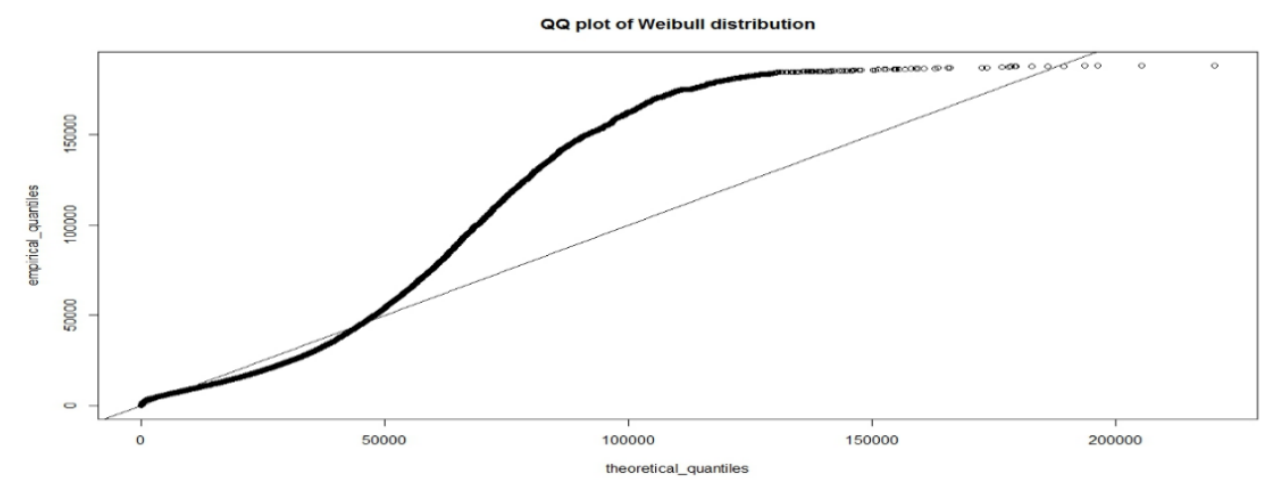

Figure 3 QQ Plot between the empirical quantiles estimated from the motor insurance data and the theoretical quantiles for the Weibull distribution with $\hat{\theta}=18058.838357$ and $\hat{\beta}=1.0196673$

Table 3 shows the values of the ultimate ruin probability for our fitted Weibull model computed through FFT. In executing this computation, the main challenge lies in determining the distribution function of its first order equilibrium distribution for it is this distribution which has to be discretized and thereafter be used as input array for executing the FFT. Since this distribution function is computed by the use of the pgamma function of $\mathrm{R}$, an element of approximation got further added to the computation. In executing the FFT, we have used central difference discretization to discretize the equilibrium distribution. The truncation parameter $M$ is chosen to be $M=655356$ and the discretization interval is taken as $\sigma=$ 0.001. The inbuilt function "fft" of the R software is used to compute the FFT. As evident from Table 3, values of ultimate ruin probability $\psi(u)$ is found to be decreasing with an increase in the initial surplus and this is intuitively logical for larger initial surplus, tends to diminish the chance of ruin, if any. Also, the values of initial surpluses which are used are solely illustrative. The actual initial surplus used by an insurance company is an internal 
decision which it might choose not to display in public.

Table 4 shows the value of ultimate ruin probability computed through the 4 moment gamma De Vylder's approximation. One of the main reasons to include it in our paper is to check the consistency of the values of $\psi(u)$ obtained for the fitted Weibull through FFT with a standard approximation method for the ultimate ruin probability. The values of $\psi(u)$ obtained through FFT and the 4 moment gamma De Vylder approximation are fairly consistent with each other. This leads us to conclude on the efficiency of FFT in computing the ultimate ruin probability for Weibull distribution. The details on the execution procedure of the 4 moment Gamma De Vylder's approximation in Xplore software is found in [3]. No attempt is made in this paper to investigate the complexity occurring in the process of computation of the integral appearing in equation (24).

Table 3. Computation of the probability of ultimate ruin for the fitted Weibull distribution with $\hat{\theta}=18058.838357$ and $\hat{\beta}=1.0196673$ through FFT

\begin{tabular}{|c|c|}
\hline $\begin{array}{c}\text { Initial Surplus } \\
(u \text { in } R s)\end{array}$ & Probability of ultimate Ruin $(\psi(u))$ \\
\hline 10 & 0.7691160 \\
\hline 20 & 0.7690002 \\
\hline 30 & 0.7688851 \\
\hline 40 & 0.7687694 \\
\hline 50 & 0.7686545 \\
\hline 60 & 0.7685389 \\
\hline 70 & 0.7684241 \\
\hline 80 & 0.7683086 \\
\hline 90 & 0.7681939 \\
\hline 100 & 0.7680785 \\
\hline 200 & 0.7669308 \\
\hline 500 & 0.7635178 \\
\hline 1000 & 0.7579297 \\
\hline
\end{tabular}


Table 4. Computation of the probability of ultimate ruin for the fitted Weibull distribution with $\hat{\theta}=18058.838357$ and $\hat{\beta}=1.0196673$ through the 4 moment Gamma De Vylder's

Approximation

\begin{tabular}{|c|c|}
\hline $\begin{array}{c}\text { Initial Surplus } \\
(u \text { in } R s)\end{array}$ & Probability of ultimate Ruin $(\psi(u))$ \\
\hline 10 & 0.76859 \\
\hline 20 & 0.76849 \\
\hline 30 & 0.76839 \\
\hline 40 & 0.76829 \\
\hline 50 & 0.76820 \\
\hline 60 & 0.76810 \\
\hline 70 & 0.76800 \\
\hline 80 & 0.76790 \\
\hline 90 & 0.76781 \\
\hline 100 & 0.76771 \\
\hline 200 & 0.76674 \\
\hline 500 & 0.76382 \\
\hline 1000 & 0.75897 \\
\hline
\end{tabular}

Table 5 displays the first two moments of the deficit at the time of ruin in case of our fitted Weibull distribution. An idea on the severity of the ruin can be obtained by the mean (first moment) of the deficit at the time of ruin. The mean of the deficit at the time of ruin is found to be decreasing with an increase in the initial surplus. This of course, is an expected trend since larger initial surpluses should decrease the severity of the ruin. The second order moment of the deficit at the time of ruin is also found to be increasing with an increase in the initial surplus. An idea on the deficit at the time of ruin is important because it guides the action to be taken for recovery in case of ruin for the insurance company.

The first two moments of the surplus prior to ruin for the claim severity distributed as our fitted Weibull are displayed in Table 6. Mean of the surplus just prior to ruin is found to be increasing with an increase in the initial surplus. The second moment of the surplus prior to ruin is also found to be increasing with an increase in the initial surplus. The importance of the surplus just prior to ruin can't be underestimated for it gives some insight on the amount of the claim causing ruin and hence guides the precautionary measures to be taken by the insurance company to maintain enough reserves so as to avoid any probable case of ruin (insolvency).

Table 7 gives the approximate value for the first moment (mean) of the time to ruin in case 
of the fitted Weibull distribution as a function of the initial surplus. The mean of the time to ruin is found to be increasing with an increase in the initial surplus. This is intuitively logical for the induction of larger surpluses should prolong the time to ruin, if ruin at all occurs. An interpretation of a typical value in Table 5 is that for the claim severity distributed as our fitted Weibull, starting with an initial surplus of Rs 500, it would on the average take 0.1029923 years for the surplus process to be less than or equal to zero for the first time thereby leading to ruin in the sense of its definition.

Table 8 gives the second moment of the time to ruin in case of the fitted Weibull. It is interesting to observe that 2 nd moment of the time to ruin tends to increase with an increase in the initial surplus. It needs further exploration to justify why the heterogeneity in the time to ruin should increase with an increase in the initial surplus.

Table 5: First two moments of the deficit at the time of ruin for the fitted Weibull distribution with $\hat{\theta}=18058.838357$ and $\hat{\beta}=1.0196673$

\begin{tabular}{|c|c|c|}
\hline $\begin{array}{c}\text { Initial Surplus } \\
(u \text { in } R s)\end{array}$ & $\begin{array}{c}\text { First Moment of the deficit } \\
\text { (in Rs) }\end{array}$ & Second moment of the deficit \\
\hline 10 & 17572.88 & 611692384 \\
\hline 20 & 17572.69 & 611682425 \\
\hline 30 & 17572.50 & 611672491 \\
\hline 40 & 17572.31 & 611662580 \\
\hline 50 & 17572.12 & 611652691 \\
\hline 60 & 17571.94 & 611642824 \\
\hline 70 & 17571.75 & 611632977 \\
\hline 80 & 17571.56 & 611623151 \\
\hline 90 & 17571.38 & 611613344 \\
\hline 100 & 17571.19 & 611603557 \\
\hline
\end{tabular}


WEIBULL DISTRIBUTION AS AN ACTUARIAL RISK MODEL: COMPUTATION OF ITS PROBABILITY OF ULTIMATE RUIN AND THE MOMENTS OF THE TIME TO RUIN, DEFICIT AT RUIN AND SURPLUS

PRIOR TO RUIN

Table 6: First two moments of the surplus prior to ruin for the fitted Weibull distribution with

$$
\hat{\theta}=18058.838357 \text { and } \hat{\beta}=1.0196673
$$

\begin{tabular}{|c|c|c|}
\hline $\begin{array}{c}\text { Initial Surplus } \\
(u \text { in } R s)\end{array}$ & $\begin{array}{c}\text { First Moment of the surplus just prior to ruin } \\
\text { (in Rs) }\end{array}$ & $\begin{array}{c}\text { Second moment of the } \\
\text { surplus just prior to ruin }\end{array}$ \\
\hline 10 & 17582.88 & 612043866 \\
\hline 20 & 17592.68 & 612385431 \\
\hline 30 & 17602.48 & 612727061 \\
\hline 40 & 17612.28 & 613068756 \\
\hline 50 & 17622.07 & 613410514 \\
\hline 60 & 17631.86 & 613752335 \\
\hline 70 & 17641.65 & 614094217 \\
\hline 80 & 17651.43 & 614436161 \\
\hline 90 & 17661.21 & 614778165 \\
\hline 100 & 17670.98 & 615120229 \\
\hline
\end{tabular}

Table 7: First moments of the time to Ruin for the fitted Weibull distribution with $\hat{\theta}=$ 18058.838357 and $\hat{\beta}=1.0196673$ with $\lambda=32.427$

\begin{tabular}{|c|c|}
\hline $\begin{array}{c}\text { Initial Surplus } \\
(u \text { in } R s)\end{array}$ & $\begin{array}{c}\text { First Moment } \\
\text { (Mean in years) }\end{array}$ \\
\hline 10 & 0.1008798 \\
\hline 20 & 0.1009229 \\
\hline 30 & 0.1009659 \\
\hline 40 & 0.1010090 \\
\hline 50 & 0.1010521 \\
\hline 60 & 0.1010951 \\
\hline 70 & 0.1011382 \\
\hline 80 & 0.1011813 \\
\hline 90 & 0.1012243 \\
\hline 100 & 0.1012674 \\
\hline 200 & 0.1016983 \\
\hline 500 & 0.1029923 \\
\hline
\end{tabular}


Table 8: Second moments of the time to Ruin for the fitted Weibull distribution with $\hat{\theta}=$

\begin{tabular}{|c|c|}
\hline $\begin{array}{l}\text { Initial Surplus } \\
\qquad(u \text { in } R s)\end{array}$ & Second Moment \\
\hline 10 & 0.03805771 \\
\hline 20 & 0.03807758 \\
\hline 30 & 0.03809744 \\
\hline 40 & 0.03811732 \\
\hline 50 & 0.03813719 \\
\hline
\end{tabular}

Table 9 shows the probability of ultimate ruin for the fitted weibull distribution under the influence of interest earnings and tax payments. As expected, the probability of ultimate ruin is found to be decreasing with an increase in the initial surplus. The initial surpluses displayed are solely for illustration. The computation for evaluating the integral stated in (63) involves numerical integration and hence there remains scope for the occurrence of error although the error margin remained as low as 1e-10. The general perception is that the interest earning should diminish the chance of ruin whereas tax payment being an outflow, should increase the chance of ruin. The computation of the probability of ultimate ruin through FFT and the 4 moment gamma De Vylder approximation, as shown in sub sections(2.3) and (2.4) has not considered the influence of interest earnings and tax payments. Comparing the values of $\psi(u)$ computed under interest earnings and tax payments with those values lead us to conclude that net impact of interest earning and tax payment is negative thereby increasing the probability of ultimate ruin in our case. It needs mentioning that the interest rate used is purely illustrative and the tax structure is extracted from Wei, 2009. A tax structure prevalent in India during the time data was collected would have been more realistic. 
Table 9: Probability of ultimate ruin for Weibull Distribution with parameters $\hat{\theta}=$ 18058.838357 and $\hat{\beta}=1.0196673$ under the tax structure given by (62) and rate of interest $\delta=0.05$.

\begin{tabular}{|c|c|}
\hline $\begin{array}{c}\text { Value of the initial surplus } u \\
\text { (in Rs) }\end{array}$ & $\psi_{\delta, \gamma}(u)$ \\
\hline 10 & 0.8534887 \\
\hline 20 & 0.8530119 \\
\hline 30 & 0.8525354 \\
\hline 40 & 0.8520591 \\
\hline 50 & 0.8515830 \\
\hline 60 & 0.8511072 \\
\hline 70 & 0.8506316 \\
\hline 80 & 0.8501563 \\
\hline 90 & 0.8496812 \\
\hline 100 & 0.8492063 \\
\hline 200 & 0.8482574 \\
\hline 500 & 0.8468357 \\
\hline 1000 & 0.8074712 \\
\hline
\end{tabular}

\section{Concluding Remark}

The computational methodologies in regards to a fitting of a Weibull distribution including the MLE estimates of its parameters and testing the goodness of fit through two EDF statistics are presented. The values of the ultimate ruin probabilities obtained for the fitted Weibull model through both the methods, namely FFT and the 4 moment Gamma De Vylder approximation are found to be fairly consistent with one another. The first moments of the time to ruin and the deficit at the time of ruin indicate trends, consistent with the behavior expected in practice. The observed trend of the first moment of the surplus prior to ruin is exhibiting a behavior contrary to what is expected and will require further probe to identify the cause behind this behavior. In case of our fitted model, under the influence of the interest rate and tax structure, the probability of ultimate ruin is found to be increasing compared to the situation when the influence of both the factors is considered to be absent. The computations occurring in the paper make use of extensive programming in $\mathrm{R}$. 
The limitations of the work include the use of a model which lacks adequate fit to the data set under consideration. The computation of some of the actuarial quantities under consideration is extremely time consuming with the executing time extending to four hours in some cases. The accumulation of error via the simultaneous numerical integrations carried out in a nested order could not be avoided. Furthermore, the values of $\psi(u)$ which were used as input for the numerical integrations aimed at obtaining the required moments are taken from the stable recursive algorithm. The inclusion of these values computed through FFT as input for the subsequent numerical integrations will be more meaningful in maintaining the coherence among the contents of the work cited in this paper.

Further extension of the work could be directed at removing the limitations of the work indicated above. Efforts can be made to furnish explanation for the behavior observed in some of the actuarial quantities. FFT can further be manipulated to compute the moments of the time to ruin and future work may also be aimed at studying the individual influence of interest earnings and the tax payments on the probability of ultimate ruin for the Weibull distribution as the underlying loss model. 


\section{References}

[1] Albrecher, H , Hipp, C(2007): Lundberg's Risk Process with tax, Blatter Der, DGVFM, 28(1), $13-28$

[2] Asmussen, S. (2000): Ruin Probabilities, World Scientific, Singapore

[3] Borak, S, Hardle, W and Lehmann, H (2005): Working with the XQC, Statistical Tools for Finance and Insurance, Eds: Cizek, P, Hardle, W and Weron, R, Springer.

[4] Bowers, N.L., Gerber, H.U., Hickman, J.C., Jones, D.A. and Nesbitt, C.J. (1998): Actuarial Mathematics, Society of Actuaries, Itasca, Illinois

[5] Burnecki, K, Mista, P and Weron, A (2003): A New De Vylder Type Approximation of the Ruin Probability in infinite Time, Research Report, HSC/03/05

[6] Burnecki, K., Misiorek, A and Weron, R. (2005): Loss Distributions, Statistical Tools For Finance and Insurance, Springer Berlin Heidelberg

[7] Cummins, J.D.; Dionne, G.; McDonald ,J.B. and Pritchett, B.M. (1990): Applications of the GB2 Family of Distributions in Modelling Insurance Losses, Insurance: Mathematics and Economics, 9, pp 257-272

[8] Dickson, D.C.M (1992): On the Distribution of surplus prior to ruin, Insurance: Mathematics and Economics 11, pp 191-207

[9] Dickson, D.C.M, Egideo dos Reis, A.D.E. (1996): On the distribution of the duration of negative surplus, Scandinavian Actuarial Journal, pp 148-164

[10] Dickson, D.C.M., Egideo dos Reis, A.D. and Waters, H.R. (1995): Some Stable (Algorithms in Ruin Theory and their Applications, ASTIN Bulletin, 25, pp 153-175

[11] Dickson, D.C.M, Waters, H.R. (2002): The Distribution of the Time to Ruin in the Classical Risk Model, ASTIN Bulletin, 32, pp 299-313

[12] Dufresne, F, Gerber, H.U. (1988): The probability and severity of ruin for combination of Exponential claim amount distribution and their translations. Insurance: Mathematics and Economics, 7, pp 75-80 
[13] Embrechts, P, Grubel, R and Pitts, S.M.(1993): Some applications of the Fast Fourier Transform algorithm in Insurance Mathematics, Statistica Neerlandica, 47, 59-75

[14] Embrechts, P., Kluppelberg, C and Mikosh, T (1997): Modelling Extremal Events for Insurance and Finance, Springer

[15] Gerber, H.U. (1979): An introduction to Mathematical Risk Theory, Homewood Richard D. Irwin Inc

[16] Gerber, H.U., Goovaerts, M, Kaas, R. (1987): On the probability and severity of ruin, ASTIN Bulletin, 17, pp 151-163

[17] Gerber, H.U, Shiu, E.S.W (1997): The joint distribution of the time of ruin, the surplus immediately before ruin and the deficit at ruin, Insurance: Mathematics and Economics, pp 129137

[18] Gerber, H.U, Shiu, E.S.W (1998): On the time value of Ruin, North American Actuarial Journal, $2, \mathrm{pp} 48-78$

[19] Goovaerts, M and De Vylder, F (1984): A Stable Recursive Algorithm for the Evaluation of Ultimate Ruin Probabilities, ASTIN Bulletin, 14, pp 53-59

[20] Grandell, J (1991): Aspects of Risk Theory, Springer, New York

[21] Grandell J, (2000): Simple Approximations of Ruin Probability, Insurance: Mathematics and Economics, 26, 157-173

[22] Grubel, R and Hermesmeir, R (1999): Computation of compound distributions I: aliasing error and Exponential tilting, ASTIN Bulletin, 29, 197-214

[23] Grubel, R and Hermesmeir, R (2000): Computation of compound distributions II: discretization errors and Richardson extrapolation, ASTIN Bulletin, 30, 309-331

[24] Gupta, A.K. and Aziz, M.A. (2015): Comonotonic Approximation for the sumof Log Unified skew normal random variables: Application in Finance and Actuarial Science, Journal of Data Science, 13, 369-384

[25] Hogg, R and Klugman, S.A. (1984): Loss Distributions, Wiley, New York

[26] Kaas, R; Goovaerts, M; Dhaene, J and Denuit, M (2008) Modern Actuarial Risk Theory, Springer 
WEIBULL DISTRIBUTION AS AN ACTUARIAL RISK MODEL: COMPUTATION OF ITS PROBABILITY

OF ULTIMATE RUIN AND THE MOMENTS OF THE TIME TO RUIN, DEFICIT AT RUIN AND SURPLUS

PRIOR TO RUIN

[27] Kleiber,C and Kotz, S (2003): Statistical size distributions in Economics and Actuarial Science, Wiley Series in Probability and Statistics

[28] Klugman, S.A., Panjer, H.H and Willmot, G.E. (1998): Loss Models: From Data to Decisions, Wiley, New York

[29] Lima, F.D.P.; Garcia ,J.M.A and Dos Reis, A.D.E. (2002): Fourier/Laplace Transforms and Ruin Probabilities, ASTIN Bulletin, 32, 91-105

[30] Lin, X.S. and Willmot, G.E. (1999): Analysis of a Defective Renewal Equation Arising in Ruin Theory, Insurance Mathematics and Economics, 25, pp 63-84

[31] Lin, X.S. and Willmot, G.E. (2000): The Moments of the Time to Ruin, the Surplus before Ruin and the Deficit at Ruin, Insurance Mathematics and Economics, 27, pp 19-44

[32] Panjer, H.H. and Willmot, G.E. (1992): Insurance Risk Models, Society of Actuaries, Schaumburg

[33] Pitts, S.M. (2006): The Fast Fourier Transform Algorithm in Ruin theory for the Classical Risk Model, HERMIS: An International Journal of Computer Mathematics and its Applications, 7, 8094.

[34] R Core Team (2013): R: A language and environment for Statistical computing, R Foundation for Statistical computing, Vienna, Austria, ISBN-3-900051-07-0, URL http:// www. R-project.org/

[35] Ross, S (2002): Simulation Academic Press, San Diego

[36] Schmidli, H. (1999): On the distribution of surplus prior to and at ruin, ASTIN Bulletin, 29, pp 227-244

[37] Stephens, M.A. (1978): On the half sample methods for goodness of fit. Journal of the Royal Statistical Society B, 40: 64-70

[38] Teimouri, M. and Gupta, A.K. (2013): On the Three Parameter Weibull Distribution Shape Parameter Estimation, Journal of Data Science, 11, 403-414.

[39] Watkins, J.A. (1999): An Algorithm for Maximum Likelihood Estimation in the three parameter Burr XII distribution, Computational Statistics and Data Analysis, 32, pp 19-27. 
[40] Wei, L (2009): Ruin Probability in the presence of interest Earnings and Tax Payments, Insurance: Mathematics and Economics, 45, 133-138

[41] Weibull, W. (1951): A Statistical Distribution Function of wide Applicability, J Appl. Mech. Trans. ASME, 18(3), pp 293-297

[42] Willmot, G.E and Lin, X.S. (1998): Exact and approximate properties of the distribution of surplus before and after ruin, Insurance: Mathematics and Economics, 23, pp 91-110 


\section{Appendix:}

\section{The Newton Raphson Method: The Multiparameter Situation}

One of the most used methods for optimization in the Multi Parameter situation in Statistics is the Newton -Raphson method which is described briefly as given below:

Assume $\theta=\left(\theta_{1}, \theta_{2}, \ldots \theta_{p}\right)^{T}$ is a s a vector of $\mathrm{p}$ (say) unknown parameters and the $\log$ likelihood of the distribution involving $\theta$ is given by $\mathrm{l}(\theta, \tilde{x})$. Then the MLE for $\theta$ are obtained by solving the equations $\mathrm{l}(\theta, \tilde{x})=0$.

Let us now define what is known as the gradient matrix and the Hessian matrix given by

The gradient matrix is given by

$$
S(\theta)=\left(\begin{array}{c}
\frac{\delta l}{\delta \theta_{1}} \\
\frac{\delta l}{\delta \theta_{2}} \\
\dot{\delta l} \\
\delta \theta_{p}
\end{array}\right)
$$

And the Hessian matrix is given by

$$
J(\theta)=\left(U_{i, j}\right)_{i, j=1,2, \ldots p}
$$

Where

$$
J_{i, j}=-\frac{\delta^{2} \mathrm{l}(\theta)}{\delta \theta_{i} \sigma \theta_{j}} .
$$

Then the iterative relationship for the multi parameter Newton Raphson method is given by

$$
\theta^{(s+1)}=\theta^{(s)}+\left[J\left(\theta^{(s)}\right)\right]^{-1} s\left(\theta^{(s)}\right)
$$

Where $\theta^{(s)}$ is the estimated value of $\theta$ at the $s^{\text {th }}$ iteration. The iteration is carried out until there is no significant difference between $\theta^{(s)}$ and $\theta^{(s+1)}$.

\section{Multi parameter Newton Raphson for Weibull distribution}

The log likelihood of the Weibull distribution is given by equation (3).

The Gradient matrix for Weibull is given by

$$
\mathrm{G}=\left[\begin{array}{l}
g_{1} \\
g_{2}
\end{array}\right]
$$

Where

$$
g_{1}=\frac{m}{\beta}-m \log \theta+S_{e}+\theta^{-\beta} \log \theta S_{0}(\beta) \text { and }
$$




$$
g_{2}=-\frac{m \beta}{\theta}+\beta \theta^{-\beta-1} S_{0}(\beta)
$$

And the Hessian matrix is given by $\mathrm{H}=\left[\begin{array}{ll}a_{11} & a_{12} \\ a_{21} & a_{22}\end{array}\right]$, where

$$
\begin{aligned}
& a_{11}=\frac{m}{\beta^{2}}+\theta^{-\beta} S_{0}(\beta)\left(\log _{e} \theta\right)^{2}-2 \theta^{-\beta} S_{1}(\beta) \log _{e} \beta+\theta^{-\beta} S_{2}(\beta) \\
& a_{12}=\frac{m}{\theta}-S_{0}(\beta)\left\{\left(1-\beta \log _{e} \theta\right) \theta^{-\beta-1}\right\}-\beta \theta^{-\beta-1} S_{1}(\beta) \\
& a_{21}=a_{12} \\
& a_{22}=\frac{\mathrm{m} \beta}{\theta^{2}}+\beta(\beta+1) \frac{1}{\theta^{\beta+1}} S_{0}(\beta)
\end{aligned}
$$

And

$$
S_{j}(t)=\sum_{r=1}^{m} d_{r}^{t}\left(\log d_{r}\right)^{j}
$$

